\title{
A connection between the maximum displacements of rogue waves and the dynamics of poles in the complex plane
}

\author{
T. Y. Liu, ${ }^{1}$ T. L. Chiu, ${ }^{1}$ P. A. Clarkson, ${ }^{2}$ and K. W. Chow ${ }^{1, a)}$ \\ ${ }^{1}$ Department of Mechanical Engineering, University of Hong Kong, Pokfulam, Hong Kong \\ ${ }^{2}$ School of Mathematics, Statistics and Actuarial Science, University of Kent, Canterbury, Kent CT2 7FS, \\ United Kingdom
}

(Received 21 August 2017; accepted 6 September 2017; published online 25 September 2017)

\begin{abstract}
Rogue waves of evolution systems are displacements which are localized in both space and time. The locations of the points of maximum displacements of the wave profiles may correlate with the trajectories of the poles of the exact solutions from the perspective of complex variables through analytic continuation. More precisely, the location of the maximum height of the rogue wave in laboratory coordinates (real space and time) is conjectured to be equal to the real part of the pole of the exact solution, if the spatial coordinate is allowed to be complex. This feature can be verified readily for the Peregrine breather (lowest order rogue wave) of the nonlinear Schrödinger equation. This connection is further demonstrated numerically here for more complicated scenarios, namely the second order rogue wave of the Boussinesq equation (for bidirectional long waves in shallow water), an asymmetric second order rogue wave for the nonlinear Schrödinger equation (as evolution system for slowly varying wave packets), and a symmetric second order rogue wave of coupled Schrödinger systems. Furthermore, the maximum displacements in physical space occur at a time instant where the trajectories of the poles in the complex plane reverse directions. This property is conjectured to hold for many other systems, and will help to determine the maximum amplitudes of rogue waves. Published by AIP Publishing. [http://dx.doi.org/10.1063/1.5001007]
\end{abstract}

\begin{abstract}
Rogue waves are surprisingly large displacements from an equilibrium or otherwise tranquil background. Such large amplitude waves obviously constitute a major risk for marine shipping and offshore structures. Since the discovery of similar entities along optical waveguides recently, intensive efforts have been invested to study such violent motions in a wide spectrum of physical disciplines, under the general category of "extreme and rare events in physics." The widely applicable nonlinear Schrödinger equation governs the evolution of slowly varying wave packets. The exact rational solution, the "Peregrine breather" localized in both space and time, has often been utilized as the simplest model of a rogue wave. Here, the locations of second order rogue waves are shown to correlate with the movement of poles in the complex plane, if the spatial variable of the exact solution is extended by analytic continuation. The same feature is demonstrated for the Boussinesq equation which models bidirectional wave motions in shallow water. This correlation between locations of maximum displacements in physical space and trajectories of poles in the complex plane is conjectured to be valid for many other dynamical systems in fluid mechanics and optics.
\end{abstract}

\section{INTRODUCTION}

Rogue waves are surprisingly large displacements from an equilibrium or otherwise tranquil background. Initial

\footnotetext{
a) Author to whom correspondence should be addressed: kwchow@hku.hk, Tel.: (852) 3917 2641. Fax: (852) 28585415.
}

interest in these large amplitude waves tends to focus on the oceanic setting. ${ }^{1}$ Since the discovery of similar entities along optical waveguides, intensive efforts have been invested to study such motions in a wide spectrum of physical disciplines, under the general category of "extreme and rare events in physics."2 The nonlinear Schrödinger equation is a widely applicable model in fluid mechanics and optics. ${ }^{3,4}$ One exact rational solution, the "Peregrine breather," is localized in both space and time. This solution has often been utilized as the simplest model of a rogue wave. ${ }^{5-9}$ Extension to discrete systems and rogue wave pairs with elevations and depressions has been performed. ${ }^{10,11}$

A benchmark entity in the theory of nonlinear waves is the soliton, which preserves its identity after collision with other solitons. ${ }^{4}$ An illuminating theoretical perspective is to describe the collisions of solitons in terms of interactions of poles by analytic continuation of the spatial or temporal coordinate to a complex variable. Such investigations had been performed for the classical Korteweg-de Vries and Boussinesq equations. ${ }^{12-14}$

The objective here is to apply this scheme of pole movements to the dynamics of rogue waves. In particular, this approach can reveal peculiar relations between the maxima of the rogue wave profiles in the physical plane and the real parts of the poles in the extended complex plane. Classical dynamical systems, e.g., Boussinesq and nonlinear Schrödinger equations, will be employed, but this feature will likely be valid for other evolution equations too. We first start by looking at a standard case, namely, the Peregrine breather for the Schrödinger equation (Sec. II). We then extend this idea to a purely real dynamical system (Boussinesq equation, Sec. III) 
and complex-valued envelope equations (coupled Schrödinger models, Sec. IV), and finally draw concluding remarks (Sec. V).

\section{ROGUE WAVES AND POLE DYNAMICS}

The nonlinear Schrödinger equation (with $\alpha$ and $\sigma$ being real parameters)

$$
i A_{t}+A_{x x}+\sigma A^{2} A^{*}=0
$$

possesses the rational solution ${ }^{5}$

$$
A=\alpha \exp \left(i \sigma \alpha^{2} t\right)\left\{1-\frac{2\left(1+2 i \sigma \alpha^{2} t\right)}{\sigma \alpha^{2}\left(x^{2}+2 \sigma \alpha^{2} t^{2}+\frac{1}{2 \sigma \alpha^{2}}\right)}\right\}
$$

which is nonsingular only for $\sigma>0$. The poles of Eq. (2) occur at

$$
x= \pm\left[2 \sigma \alpha^{2} t^{2}+1 /\left(2 \sigma \alpha^{2}\right)\right]^{1 / 2} i
$$

and trajectories in the complex $x$ plane are illustrated in Fig. 1. The maximum height of the rogue wave is located at $x=0$ in laboratory coordinates (real $x$ and $t$ ). If $x$ is allowed to be complex, the real part of the poles of the exact solution vanishes too [Eq. (3)]. This leads us to the following conjecture.

\section{A. Conjecture}

"The locations of the maximum height of a rogue wave in laboratory coordinates (real space and time) will be equal to the real part of the poles of the exact solution by analytic continuation, if the spatial coordinate is allowed to be complex."

Besides the formulation outlined above, the conjecture can also be verified analytically for the lowest order rogue wave of the derivative nonlinear Schrödinger equation. ${ }^{15}$ Preliminary numerical testing has been performed for the symmetric second order rogue waves of the single component nonlinear Schrödinger equation, and the results are very encouraging. ${ }^{15}$ The goal here is to conduct further numerical tests using a real evolution system and also asymmetric as

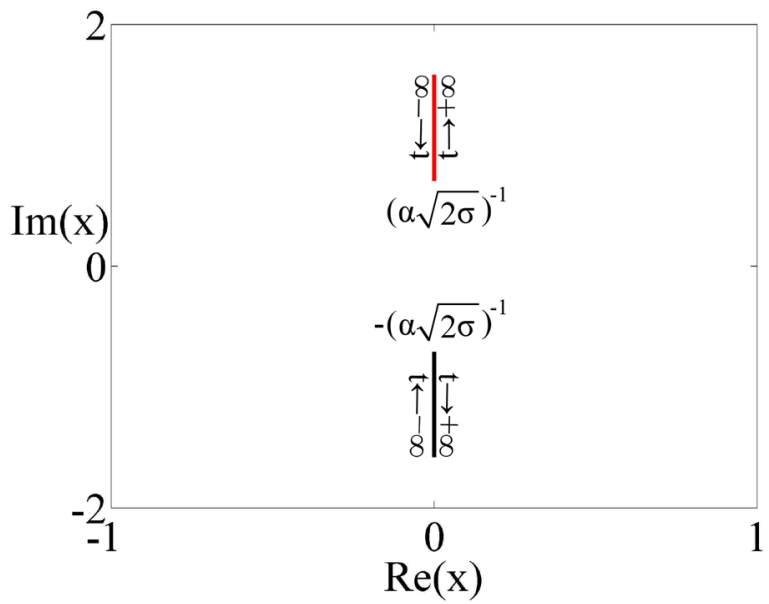

FIG. 1. The motion of the two poles of the lowest order rogue wave of the nonlinear Schrödinger equation in the complex " $x$ " plane [Eq. (3)]. well as symmetric rogue waves for (coupled) complexvalued evolution equations. Such numerical tests can be justified from the following perspective. For a simple case like Eq. (2), elementary calculus can be applied to ascertain that the turning points of $|A|^{2}$ occur along $t=0$. In more complicated scenarios discussed below, the turning points may occur off the axes in the $x, t$ plane, and the algebra then becomes oppressive. Remarkably, this information from pole dynamics might still reveal characteristics of the wave profiles for a large class of evolution systems.

\section{THE BOUSSINESQ EQUATION}

\section{A. Analytical formulation}

Physically, the Boussinesq equation describes bidirectional wave propagation in shallow water ${ }^{16,17}$

$$
u_{t t}+u_{x x}-\left(u^{2}\right)_{x x}-\frac{1}{3} u_{x x x x}=0,
$$

where $u$ typically denotes the free surface displacement or the horizontal velocity. A "tau function" or bilinear transformation

$$
u_{n}=2\left(\log f_{n}\right)_{x x}
$$

converts the issue of solving for rational solutions $u_{n}$ of Eq. (4) to a process in identifying suitable polynomials for the function $f_{n}$, where $n$ is the order of the rogue waves. ${ }^{16,17}$ For the lowest order rogue wave $(n=1)$

$$
\begin{aligned}
& f_{1}=x^{2}+t^{2}+1, \quad u_{1}=2\left(\log f_{1}\right)_{x x} \\
& u_{1}=4\left(1-x^{2}+t^{2}\right) /\left(x^{2}+t^{2}+1\right)^{2}
\end{aligned}
$$

the conjecture holds trivially as the physical maximum for $u_{1}$ occurs at $x=t=0$, and the poles of $u_{1}$ will have zero real parts. The second order rogue wave is generated by taking this auxiliary function $f_{2}$ to be

$$
\begin{aligned}
f_{2}(x, t)= & x^{6}+\left(3 t^{2}+\frac{25}{3}\right) x^{4}+\left(3 t^{4}+30 t^{2}-\frac{125}{9}\right) x^{2} \\
& +t^{6}+\frac{17}{3} t^{4}+\frac{475}{9} t^{2}+\frac{625}{9}
\end{aligned}
$$

with increasing algebraic complexity for the higher order rogue waves.

A more intriguing case is to look into the generalized higher order rogue waves (with additional free parameters $\alpha$ and $\beta$ ) defined by

$$
\begin{aligned}
F_{n+1}(x, t ; \alpha, \beta)= & f_{n+1}(x, t)+2 \alpha t P_{n}(x, t)+2 \beta x Q_{n}(x, t) \\
& +\left(\alpha^{2}+\beta^{2}\right) f_{n-1}(x, t),
\end{aligned}
$$

where $f_{n}, P_{n}(x, t)$ and $Q_{n}(x, t)$ are polynomials in $x$ and $t .^{16,17}$ For simplicity, we just concentrate on the second order rogue wave in this work where

$$
P_{1}(x, t)=3 x^{2}-t^{2}+\frac{5}{3}, \quad Q_{1}(x, t)=x^{2}-3 t^{2}-\frac{1}{3},
$$




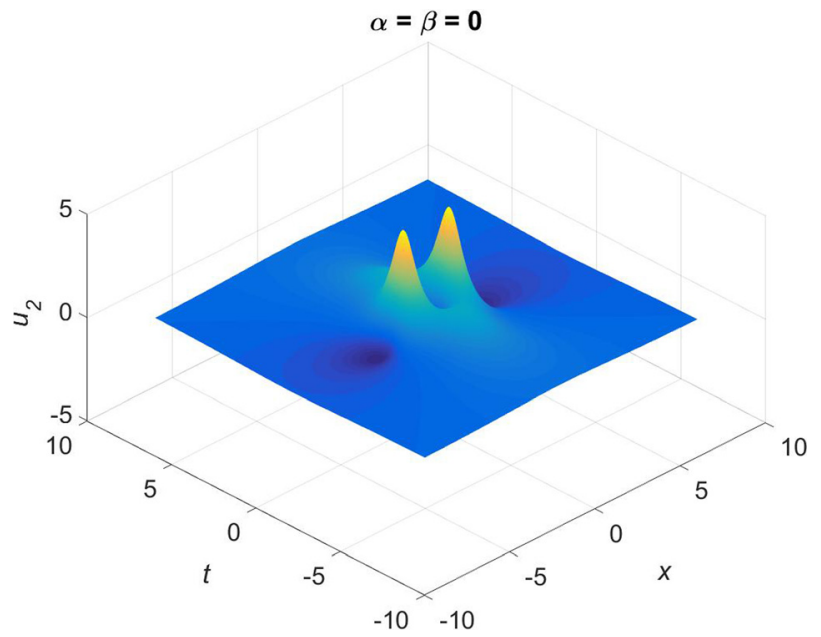

FIG. 2. Amplitude of the wave envelope $u_{2}$ versus $x$ and $t$ for the second order rogue wave of the Boussinesq equation, $\alpha=\beta=0$.

and $f_{0}=0$ (Figs. 2 and 3). To test the validity of the conjecture outlined in Sec. II, numerical evidence is presented in the following subsection (Table I and Fig. 4).

\section{B. Numerical evidence}

The trajectories of the poles reverse direction at a time instant $t$, which physically corresponds to the occurrence of maximum displacement (Fig. 4).

\section{THE NONLINEAR SCHRÖDINGER EQUATIONS: ASYMMETRIC ROGUE WAVES AND COUPLED SYSTEMS}

The evolution of slowly varying wave packets governed by complex-valued nonlinear Schrödinger equations provides yet another encouraging sign for the validity of the conjecture. The lowest order rogue wave is discussed in Sec. II, while the second order one is addressed in our earlier paper. ${ }^{15}$ Here, we focus on more complicated cases, namely, asymmetric rogue waves and the occurrence of such large amplitude modes for coupled systems.

\section{A. Asymmetric rogue waves}

For the classical nonlinear Schrödinger equation

$$
i q_{t}+q_{x x}+2 q^{2} q^{*}=0,
$$

an asymmetric rogue wave is given by ${ }^{18}$

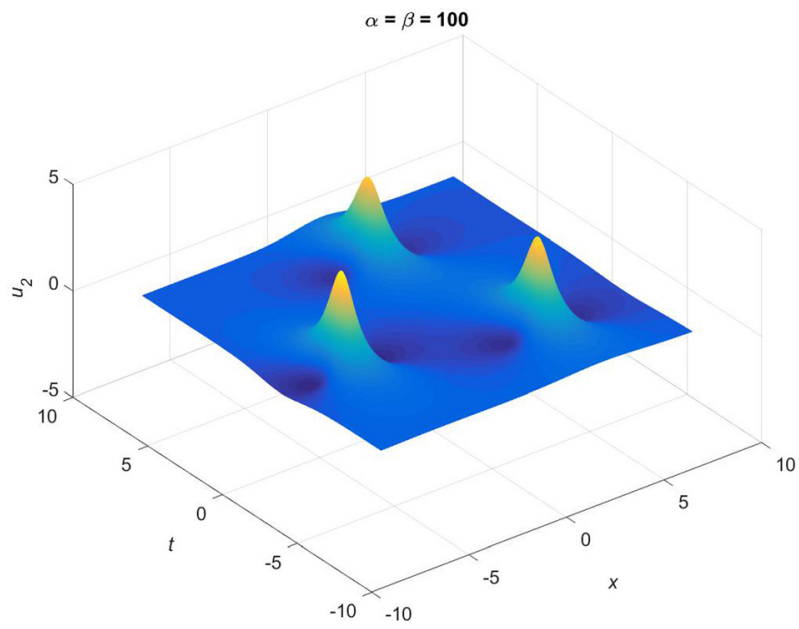

FIG. 3. Amplitude of the wave envelope $u_{2}$ versus $x$ and $t$ for the second order rogue wave of the Boussinesq equation, $\alpha=\beta=100$.

$$
\begin{gathered}
q=\left(1+\frac{F_{2}}{H_{2}}\right) e^{2 i t}, \\
F_{2}=24 i\left(12 t-32 t^{3}-12 x t+16 x^{3} t+64 t^{3} x\right. \\
-8 x^{4} t-64 x^{2} t^{3}-128 t^{5}+48 i x^{2} t^{2}-4 i x^{3} \\
\left.+6 i x^{2}+160 i t^{4}-48 i x t^{2}+48 i t^{2}+2 i x^{4}-3 i x\right), \\
H_{2}=1024 t^{6}+768 x^{2} t^{4}-768 t^{4} x+1920 t^{4}+192 x^{4} t^{2} \\
-384 x^{3} t^{2}+288 x t^{2}+288 t^{2}+16 x^{6}-48 x^{5} \\
+72 x^{4}-72 x^{3}+72 x^{2}-36 x+9 .
\end{gathered}
$$

This solution is asymmetric in the $x, t$ plane as both odd and even powers of $x$ and $t$ occur (Fig. 5). The main local maximum of the rogue wave in the physical (real) $x, t$ space is located at

$$
x=0.372, \quad t=0
$$

If $x$ in Eq. (9c) is regarded as complex, the poles of $q$ (or zeros of $\mathrm{H}_{2}$ ) at $t=0$ are located at

\begin{tabular}{|c|c|c|c|}
\hline$\alpha$ & $\beta$ & Locations of the maximum (maxima) of $u_{2}$ in the physical space with real $x$ & Location of the pole(s) of $u_{2}$ (or zeros of $f_{2}$ ) with complex $x$ \\
\hline 0 & 0 & $x= \pm 1.340, t=0$ & $t=0$ Poles located at $x=1.340 \pm 0.893 i$ and $-1.340 \pm 0.893 i$ \\
\hline 100 & 100 & $\begin{array}{l}\text { (a) } x=3.494, t=-3.514 \\
\text { (b) } x=-4.857, t=-1.257 \\
\text { (c) } x=1.260, t=4.932\end{array}$ & $\begin{array}{l}\text { (a) } t=-3.514 \text { Poles located at } x=3.494 \pm 1.003 i \\
\text { (b) } t=-1.257 \text { Poles located at } x=-4.857 \pm 0.960 i \\
\text { (c) } t=4.932 \text { Poles located at } x=1.260 \pm 1.045 i\end{array}$ \\
\hline 100 & 10 & $\begin{array}{l}\text { (a) } x=3.737, t=-2.274 \\
\text { (b) } x=-3.894, t=-2.019 \\
\text { (c) } x=0.142, t=4.555\end{array}$ & $\begin{array}{l}\text { (a) } t=-2.274 \text { Poles located at } x=3.737 \pm 0.975 i \\
\text { (b) } t=-2.019 \text { Poles located at } x=-3.894 \pm 0.968 i \\
\text { (c) } t=4.555 \text { Poles located at } x=0.142 \pm 1.063 i\end{array}$ \\
\hline
\end{tabular}

- $-0.142 \pm 1.017 i$

- $0.373 \pm 0.314 i$,

- $1.268 \pm 0.797 i$.

Again, the spatial location of the physical maximum is remarkably close to the real part of one of the poles.

The trajectories of the poles, namely, locations of zeros of $\mathrm{H}_{2}$ of Eq. (9c) provide supporting evidence (Fig. 6). There

TABLE I. Comparison of the locations of maximum displacements and the locations of poles for the Boussinesq equation. 


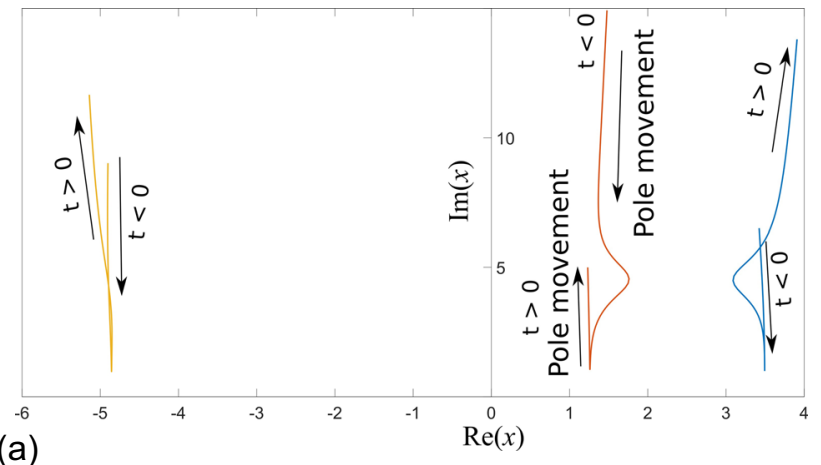

(a)

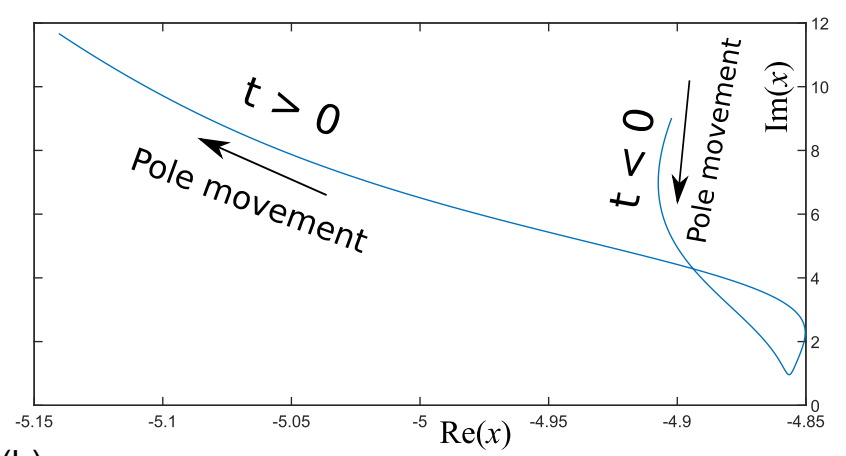

(b)

FIG. 4. Trajectories of the poles for different instants of time (t): (a) Full trajectories for all three pairs of poles for Eq. (7b); (b) A high resolution plot showing the turning point of the trajectory at $\operatorname{Re}(x)=-4.857$ (at $t=-1.257$ ).

are six branches as there are six complex roots for the sixth degree polynomial. The complex numbers where these trajectories reverse in direction have real parts very close to the spatial locations of the maximum amplitude in the physical space ( $\approx 0.373$ for the present choice of parameters).

\section{B. Coupled nonlinear Schrödinger equations}

The coupled system for the evolution of slowly varying envelopes $A, B$

$$
\begin{aligned}
& i A_{t}+A_{x x}+\sigma\left(|A|^{2}+|B|^{2}\right) A=0, \\
& i B_{t}+B_{x x}+\sigma\left(|A|^{2}+|B|^{2}\right) B=0
\end{aligned}
$$

provides an elegant analytical model for many scientific applications ( $\sigma=$ a real parameter), e.g., light polarization in

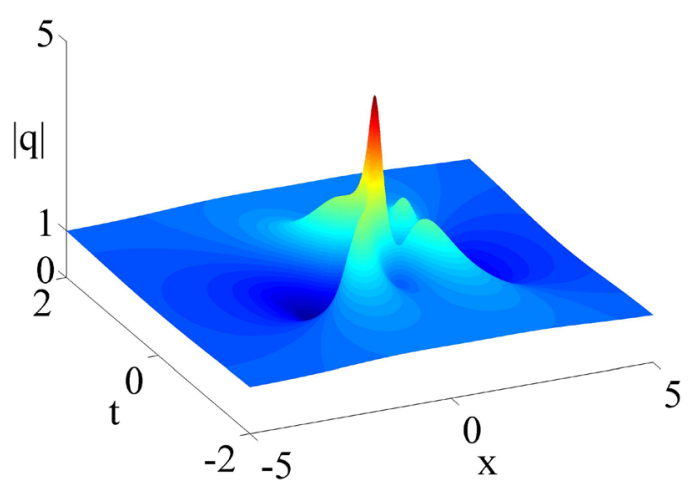

FIG. 5. Amplitude of the wave envelope $|q|$ versus $x$ and $t$ for an asymmetric rogue wavefield.

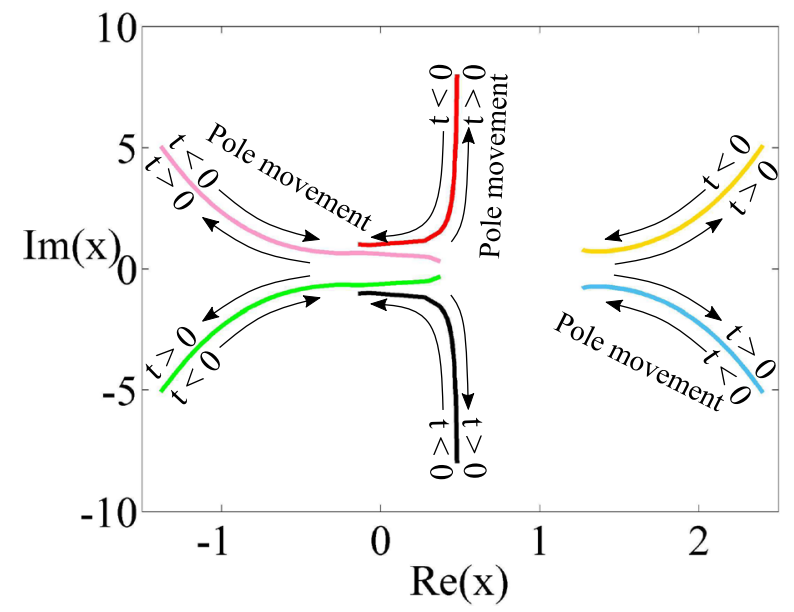

FIG. 6. The trajectories of the poles of the asymmetric rogue wave for different values of (time) $t$.

media with random birefringence. ${ }^{19,20}$ Different families of second order rogue waves have been derived. To illustrate the validity of the conjecture, only one particular case is considered here and others will be left for future studies. ${ }^{19}$ It is convenient to define the transformation

$$
A=\rho \exp \left(i k x-i \omega_{1} t\right) \frac{g}{f}, \quad B=\rho \exp \left(-i k x-i \omega_{2} t\right) \frac{h}{f}
$$

with $\rho$ being the amplitude, $k$ and $-k$ the wave numbers, and $\omega_{1}=\omega_{2}=k^{2}-2 \sigma \rho^{2}$ the frequencies of the background plane waves. In general, $\omega_{1} \neq \omega_{2}$, if there is a group velocity mismatch or a difference in background amplitudes. Rogue waves can be derived by the Hirota bilinear transform, and details are given in our earlier work. ${ }^{21}$ For simplicity, we shall focus on $\sigma=1, \rho=1$, and $k=1$, and lengthy expressions for $f, g$, and $h$ are given in the Appendix. The location of the maximum in the physical $x, t$ space occurs at

$x=-0.586, \quad t=-0.188$ and $x=0.586, \quad t=0.188$.

At $t= \pm 0.188$, the zero of the function $f$ for a complex variable $x$ is situated at

$$
-0.587 \pm 0.705 i, \quad 0.587 \pm 0.705 i .
$$

Again, we observe a remarkable coincidence. The wave profile is illustrated in Fig. 7. The complex numbers where trajectories of the poles reverse direction have real parts \pm 0.587 , remarkably close to the spatial locations of the maximum displacements (Fig. 8).

\section{CONCLUSIONS}

The locations of maximum displacements of rogue waves in the physical space might be closely related to the trajectories of the poles of the exact solution, assuming that the spatial variable is extended to the complex plane by analytic continuation. At the time instant of maximum displacement, the real part of the pole is identical or numerically 


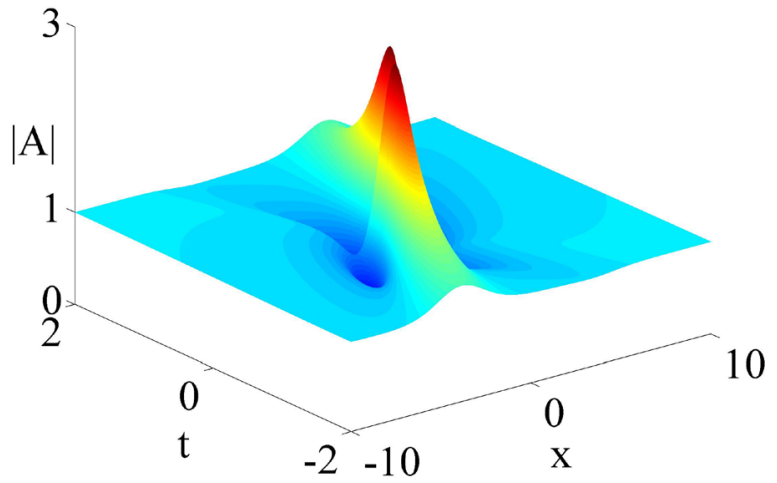

FIG. 7. Amplitude of the wave envelope $|q|$ versus $x$ and $t$ for a symmetric second order rogue wave for the coupled system.

very close to the spatial location in the physical space. Examples used here include the second order rogue waves of the Boussinesq equation, as well as the asymmetric form of the single component Schrödinger equation and the symmetric form of the coupled Schrödinger system. We conjecture that this property will hold for many other integrable systems too. A similar conjecture concerning breathers will need consideration of a periodic sequence of poles, as trigonometric and hyperbolic functions are involved. Such issues will be left for future studies.

Another peculiar feature is that the trajectories of the poles reverse direction at the time instant of the occurrence of maximum displacements in the physical space. All these properties are especially intriguing, given that the trajectories of the poles arise only from a portion of the exact solution, and not from the whole mathematical structure of the exact solution itself. If the conjecture proposed here is valid, there would be an alternative path in finding the locations and magnitude of the maximum displacements of rogue waves. However, we should also make a note of caution. In evolution systems tested so far, occasionally small discrepancies between locations of maxima and pole trajectories may exist. Naturally, we do not expect such a reasonably simple conjecture to work for all integrable systems universally. The search for more elaborate constraints for this conjecture to apply will be the goals in the near future.

Further investigations and verifications in the future would definitely be necessary. Obvious targets will be parallel calculations for the third order rogue waves and other evolution systems like the derivative nonlinear Schrödinger equations. Additional physical effects might introduce

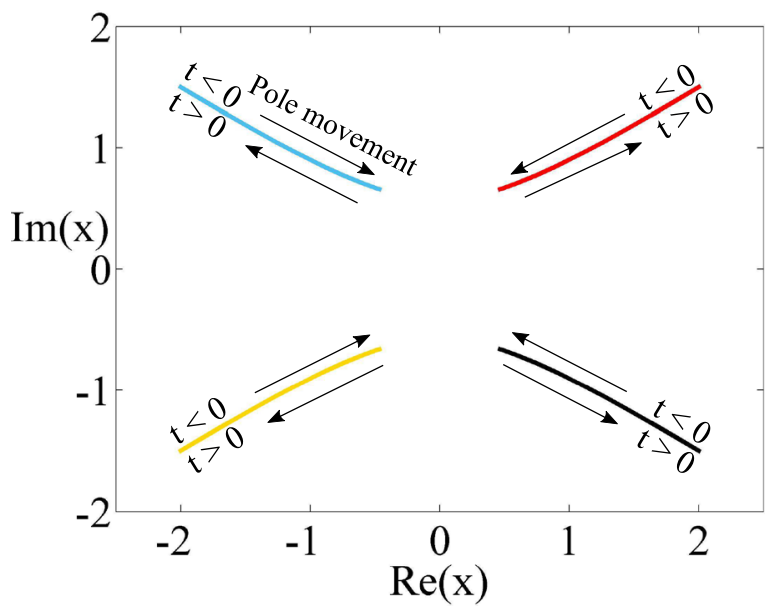

FIG. 8. The trajectories of the poles of the second order rogue wave for different values of (time) $t$.

further complexity, e.g., delayed feedback control associated with the Lugiato-Lefever equation relevant to optical cavity dynamics. ${ }^{22}$ Rogue waves occur as large intensity pulses in the transverse directions in a resonant cavity, with a governing system consisting of a complex valued envelope with a delay term. It is not known if pole dynamics introduced here can be applicable. ${ }^{23}$ Conceptually, extensions to problems with higher dimensions will present challenging issues. These increased degrees of freedom might result from transverse diffraction in two spatial dimensions or chromatic dispersion coefficient of either positive or negative sign. ${ }^{24}$ Such efforts will lead to fruitful works.

\section{ACKNOWLEDGMENTS}

Partial financial support has been provided by the Research Grants Council Contract No. HKU17200815.

\section{APPENDIX: SECOND ORDER ROGUE WAVES}

If the complex parameter $\zeta=a+i b$ is the leading order approximation in the long wave expansion for the quartic dispersion relation $\left(S=\sigma \rho^{2}\right.$ measures the amplitude and the cubic nonlinearity) of the breather/rogue wave mode of Eqs. (10) and $(11)^{21}$

$$
\zeta^{4}+4\left(S-2 k^{2}\right) \zeta^{2}+16 k^{2}\left(k^{2}+S\right)=0, \quad \zeta=a+i b,
$$

then, the rogue wave is given by the complicated expressions:

$$
f=\frac{1}{a^{4} b^{4}\left(a^{2}+b^{2}\right)^{2}}\left[\begin{array}{c}
a^{4} b^{4}\left(a^{2}+b^{2}\right)^{2} x^{4}+2 a^{4} b^{4}\left(-a^{6}-a^{4} b^{2}+a^{2} b^{4}+b^{6}\right) x^{2} t^{2} \\
+2 a^{2} b^{2}\left(a^{6}-b^{6}\right) x^{2}+a^{4} b^{4}\left(a^{2}+b^{2}\right)^{4} t^{4} \\
+2 a^{2} b^{2}\left(a^{8}+5 a^{6} b^{2}+5 a^{2} b^{6}+b^{8}\right) t^{2} \\
+a^{8}+2 a^{6} b^{2}+3 a^{4} b^{4}+2 a^{2} b^{6}+b^{8} \\
g_{n}
\end{array}\right],
$$




$$
\begin{aligned}
& g_{n}=-a^{4} b^{4}\left(a^{2}+b^{2}\right)^{2}\left[(a+2 k)^{2}+b^{2}\right]\left[(a-2 k)^{2}+b^{2}\right] x^{4}-16 a^{4} b^{4} k i\left(a^{2}+b^{2}\right)^{2}\left(-a^{2}+b^{2}+4 k^{2}\right) x^{3} \\
& +2 a^{4} b^{4}\left(a^{2}-b^{2}\right)\left(a^{2}+b^{2}\right)^{2}\left[(a+2 k)^{2}+b^{2}\right]\left[(a-2 k)^{2}+b^{2}\right] x^{2} t^{2}+8 a^{4} b^{4} i\left(a^{2}+b^{2}\right)^{2}\left[\left(a^{2}+b^{2}\right)^{2}-4 k^{2}\left(a^{2}-b^{2}\right)\right] x^{2} t \\
& +2 a^{2} b^{2}\left[\begin{array}{c}
\left(-a^{10}-6 a^{8} b^{2}-5 a^{6} b^{4}+5 a^{4} b^{6}+6 a^{2} b^{8}+b^{10}\right) \\
+8 k^{2}\left(a^{8}+5 a^{6} b^{2}+12 a^{4} b^{4}+5 a^{2} b^{6}+b^{8}\right) \\
-16 k^{4}\left(a^{6}-b^{6}\right)
\end{array}\right] x^{2}-16 a^{4} b^{4} k i\left(a^{2}+b^{2}\right)^{2}\left[a^{4}-6 a^{2} b^{2}+b^{4}-4 k^{2}\left(a^{2}-b^{2}\right)\right] x t^{2} \\
& +64 a^{4} b^{4} k\left(a^{2}-b^{2}\right)\left(a^{2}+b^{2}\right)^{2} x t+16 a^{2} b^{2} k i\left(a^{4}+a^{2} b^{2}+b^{4}\right)\left[\left(a^{2}+b^{2}\right)^{2}-4 k^{2}\left(a^{2}-b^{2}\right)\right] x \\
& -a^{4} b^{4}\left(a^{2}+b^{2}\right)^{4}\left[(a+2 k)^{2}+b^{2}\right]\left[(a-2 k)^{2}+b^{2}\right] t^{4}+8 a^{4} b^{4} i\left(a^{2}+b^{2}\right)^{4}\left(-a^{2}+b^{2}+4 k^{2}\right) t^{3} \\
& -2 a^{2} b^{2}\left[\begin{array}{c}
\left(a^{12}-5 a^{10} b^{2}-5 a^{8} b^{4}+2 a^{6} b^{6}-5 a^{4} b^{8}-5 a^{2} b^{10}+b^{12}\right) \\
+8 k^{2}\left(-a^{10}-2 a^{8} b^{2}+7 a^{6} b^{4}-7 a^{4} b^{6}+2 a^{2} b^{8}+b^{10}\right) \\
+16 k^{4}\left(a^{8}+5 a^{6} b^{2}+5 a^{2} b^{6}+b^{8}\right)
\end{array}\right] t^{2}+8 a^{2} b^{2} i\left[\left(-a^{10}+a^{6} b^{4}-a^{4} b^{6}+b^{10}\right)\right. \\
& \left.+4 k^{2}\left(a^{8}+5 a^{6} b^{2}+5 a^{2} b^{6}+b^{8}\right)\right] t-\left(a^{4}+a^{2} b^{2}+b^{4}\right)\left[\begin{array}{c}
\left(a^{8}-5 a^{6} b^{2}-12 a^{4} b^{4}-5 a^{2} b^{6}+b^{8}\right) \\
+8 k^{2}\left(-a^{6}-4 a^{4} b^{2}+4 a^{2} b^{4}+b^{6}\right) \\
+16 k^{4}\left(a^{4}+a^{2} b^{2}+b^{4}\right)
\end{array}\right] \\
& h=\frac{h_{n}}{-a^{4} b^{4}\left(a^{2}+b^{2}\right)^{2}\left[(a+2 k)^{2}+b^{2}\right]\left[(a-2 k)^{2}+b^{2}\right]}, \\
& h_{n}=-a^{4} b^{4}\left(a^{2}+b^{2}\right)^{2}\left[(a+2 k)^{2}+b^{2}\right]\left[(a-2 k)^{2}+b^{2}\right] x^{4}+16 a^{4} b^{4} k i\left(a^{2}+b^{2}\right)^{2}\left(-a^{2}+b^{2}+4 k^{2}\right) x^{3} \\
& +2 a^{4} b^{4}\left(a^{2}-b^{2}\right)\left(a^{2}+b^{2}\right)^{2}\left[(a+2 k)^{2}+b^{2}\right]\left[(a-2 k)^{2}+b^{2}\right] x^{2} t^{2}+8 a^{4} b^{4} i\left(a^{2}+b^{2}\right)^{2}\left[\left(a^{2}+b^{2}\right)^{2}-4 k^{2}\left(a^{2}-b^{2}\right)\right] x^{2} t \\
& +2 a^{2} b^{2}\left[\begin{array}{c}
\left(-a^{10}-6 a^{8} b^{2}-5 a^{6} b^{4}+5 a^{4} b^{6}+6 a^{2} b^{8}+b^{10}\right) \\
+8 k^{2}\left(a^{8}+5 a^{6} b^{2}+12 a^{4} b^{4}+5 a^{2} b^{6}+b^{8}\right) \\
-16 k^{4}\left(a^{6}-b^{6}\right)
\end{array}\right] x^{2}+16 a^{4} b^{4} k i\left(a^{2}+b^{2}\right)^{2}\left[a^{4}-6 a^{2} b^{2}+b^{4}-4 k^{2}\left(a^{2}-b^{2}\right)\right] x t^{2} \\
& -64 a^{4} b^{4} k\left(a^{2}-b^{2}\right)\left(a^{2}+b^{2}\right)^{2} x t-16 a^{2} b^{2} k i\left(a^{4}+a^{2} b^{2}+b^{4}\right)\left[\left(a^{2}+b^{2}\right)^{2}-4 k^{2}\left(a^{2}-b^{2}\right)\right] x \\
& -a^{4} b^{4}\left(a^{2}+b^{2}\right)^{4}\left[(a+2 k)^{2}+b^{2}\right]\left[(a-2 k)^{2}+b^{2}\right] t^{4}+8 a^{4} b^{4} i\left(a^{2}+b^{2}\right)^{4}\left(-a^{2}+b^{2}+4 k^{2}\right) t^{3} \\
& -2 a^{2} b^{2}\left[\begin{array}{c}
\left(a^{12}-5 a^{10} b^{2}-5 a^{8} b^{4}+2 a^{6} b^{6}-5 a^{4} b^{8}-5 a^{2} b^{10}+b^{12}\right) \\
+8 k^{2}\left(-a^{10}-2 a^{8} b^{2}+7 a^{6} b^{4}-7 a^{4} b^{6}+2 a^{2} b^{8}+b^{10}\right) \\
+16 k^{4}\left(a^{8}+5 a^{6} b^{2}+5 a^{2} b^{6}+b^{8}\right)
\end{array}\right] t^{2}+8 a^{2} b^{2} i\left[\left(-a^{10}+a^{6} b^{4}-a^{4} b^{6}+b^{10}\right)\right. \\
& \left.+4 k^{2}\left(a^{8}+5 a^{6} b^{2}+5 a^{2} b^{6}+b^{8}\right)\right] t-\left(a^{4}+a^{2} b^{2}+b^{4}\right)\left[\begin{array}{c}
\left(a^{8}-5 a^{6} b^{2}-12 a^{4} b^{4}-5 a^{2} b^{6}+b^{8}\right) \\
+8 k^{2}\left(-a^{6}-4 a^{4} b^{2}+4 a^{2} b^{4}+b^{6}\right) \\
+16 k^{4}\left(a^{4}+a^{2} b^{2}+b^{4}\right)
\end{array}\right] .
\end{aligned}
$$

${ }^{1}$ K. Dysthe, H. E. Krogstad, and P. Müller, "Oceanic rogue waves," Annu. Rev. Fluid Mech. 40, 287 (2008).

${ }^{2}$ M. Onorato, S. Residori, U. Bortolozzo, A. Montina, and F. T. Arecchi, "Rogue waves and their generating mechanisms in different physical contexts," Phys. Rep. 528, 47 (2013).

${ }^{3}$ A. D. D. Craik, Wave Interactions and Fluid Flows (Cambridge University Press, 1985).

${ }^{4}$ Y. S. Kivshar and G. P. Agrawal, Optical Solitons: From Fibers to Photonic Crystals (Academic Press, 2003).

${ }^{5}$ D. H. Peregrine, "Water-waves, non-linear Schrödinger-equations and their solutions," J. Aust. Math. Soc. B 25, 16 (1983).

${ }^{6}$ A. Ankiewicz, P. A. Clarkson, and N. Akhmediev, "Rogue waves, rational solutions, the patterns of their zeros and integral relations," J. Phys. A: Math. Theor. 43, 122002 (2010).
${ }^{7}$ Y. Ohta and J. Yang, "General high-order rogue waves and their dynamics in the nonlinear Schrödinger equation," Proc. R. Soc. A 468, 1716 (2012).

${ }^{8}$ R. H. J. Grimshaw and A. Tovbis, "Rogue waves: Analytical predictions," Proc. R. Soc. A 469, 20130094 (2013).

${ }^{9}$ N. V. Priya and M. Senthilvelan, "On the characterization of breather and rogue wave solutions and modulation instability of coupled generalized nonlinear Schrodinger equations," Wave Motion 54, 125 (2015).

${ }^{10}$ X. Y. Wen, Z. Yan, and B. A. Malomed, "Higher-order vector discrete rogue-wave states in the coupled Ablowitz-Ladik equations: Exact solutions and stability," Chaos 26, 123110 (2016).

${ }^{11}$ E. Yomba and G. A. Zakeri, "Rogue-pair and dark-bright-rogue waves of the coupled nonlinear Schrödinger equations from inhomogeneous femtosecond optical fibers," Chaos 26, 083115 (2016). 
${ }^{12} \mathrm{~K}$. Konno and H. Ito, "Nonlinear interactions between solitons in complex t-plane. I,” J. Phys. Soc. Jpn. 56, 897 (1987).

${ }^{13} \mathrm{~K}$. Konno, "Nonlinear interactions between solitons in complex t-plane. II,” J. Phys. Soc. Jpn. 56, 1334 (1987).

${ }^{14} \mathrm{M}$. Kovalyov, "On the structure of the two-soliton interaction for the Korteweg-de Vries equation," J. Diff. Equations 152, 431 (1999).

${ }^{15}$ T. L. Chiu, T. Y. Liu, H. N. Chan, and K. W. Chow, "The dynamics and evolution of poles and rogue waves for nonlinear Schrödinger equations," Commun. Theor. Phys. 68, 290 (2017).

${ }^{16}$ A. Ankiewicz, A. P. Bassom, P. A. Clarkson, and E. Dowie, "Conservation laws and integral relations for the Boussinesq equation," Stud. Appl. Math. 139, 104 (2017).

${ }^{17}$ P. A. Clarkson and E. Dowie, "Rational solutions of the Boussinesq equation and applications to rogue waves," Trans. Math. Appl. (to be published); e-print arXiv:1609.00503.
${ }^{18}$ J. He, L. Guo, Y. Zhang, and A. Chabchoub, "Theoretical and experimental evidence of non-symmetric doubly localized rogue waves," Proc. Roy. Soc. A 470, 20140318 (2014).

${ }^{19}$ J. K. Yang, "Suppression of Manakov soliton interference in optical fibers," Phys. Rev. E 65, 036606 (2002).

${ }^{20}$ V. S. Gerdjikov, M. D. Todorov, and A. V. Kyuldjiev, "Adiabatic interactions of Manakov solitons - Effects of cross-modulation,” Wave Motion 71, 71 (2017).

${ }^{21} \mathrm{H}$. N. Chan and K. W. Chow, "Rogue waves for an alternative system of coupled Hirota equations: Structural robustness and modulation instabilities," Stud. Appl. Math. 139, 78 (2017).

${ }^{22} \mathrm{M}$. Tlidi and K. Panajotov, "Two-dimensional dissipative rogue waves due to time-delayed feedback in cavity nonlinear optics," Chaos 27, 013119 (2017).

${ }^{23} \mathrm{M}$. Tlidi and K. Panajotov, "Rogue waves induced by delayed feedback in cavity nonlinear optics," J. Opt. 18, 063001 (2016).

${ }^{24}$ K. Panajotov, M. G. Clerc, and M. Tlidi, "Spatiotemporal chaos and twodimensional dissipative rogue waves in Lugiato-Lefever model," Eur. Phys. J. D 71, 176 (2017). 